\title{
Definición de un sistema experto para mejorar la gestión de inventarios: estudio de caso
}

\section{An expert system for stock replenishment improvement: a case study}

\author{
Ander Errasti ', Claudia Chackelson ' y Raúl Poler² \\ ' Dpto. de Organización Industrial.TECNUN. Escuela Superior de Ingenieros. \\ Universidad de Navarra. P. de Manuel Lardizábal . San Sebastián. \\ ${ }^{2}$ Grupo de Investigación (CIGIP).Universidad Politécnica de Valencia. \\ aerrasti@tecnun.es cchakelson@tecnun.espoler@cigip.upv.es
}

Fecha de recepción: 17-8-2010

Fecha de aceptación: 28-2-2011

\begin{abstract}
Resumen: La mejora del nivel de servicio y la reducción de inventarios en plataformas logísticas y centros de distribución, puede ayudar a mejorar la competitividad de empresas que suministran sus productos contra almacén. Este trabajo presenta el desarrollo y la implementación de un sistema experto que, mediante una clasificación ABC/XYZ de las referencias, pronostica las necesidades de producto terminado y sugiere una estrategia de aprovisionamiento para lograr alcanzar el nivel de servicio objetivo y ajustar el stock. Se detallan los resultados de un estudio de caso realizado en una empresa del sector de electrodomésticos para verificar la utilidad del sistema
\end{abstract}

Palabras clave: gestión de inventarios, previsión de demanda, clasificación ABC/XYZ, estudio de caso.

\begin{abstract}
Service level enhancement and stock reduction in distribution centers could aid to reinforce competitiveness when make to stock production systems are implemented. In this paper an inventory replenishment expert system with the objectives of improving quality service and reducing holding costs is proposed. The inventory replenishment expert system, based on an $A B C-X Y Z$ analysis and time series forecasting techniques, proposes the most effective replenishment strategy for each product category. Results of a case study developed in a manufacturer of home appliances are also shown, with the propose of testing the system utility.
\end{abstract}

Keywords: inventory management; demand forecasting, $A B C / X Y Z$ analysis, case study.

\section{Introducción}

Aunque existen empresas que consiguen realizar envíos directos de plantas productivas a cliente final, la gran mayoría requiere de una red de distribución en la cual gestionen el suministro de productos a clientes total o parcialmente contra almacén. Esta necesidad puede deberse, entre otras razones, al menor tiempo de respuesta al cliente frente al periodo de maduración fabril, la necesidad de regular flujos de suministro y producción o a la necesidad de satisfacer pedidos de cliente multiproducto con variantes fuentes de suministro (Christopher, 2004).

La gestión de los centros de almacenaje y distribución, se ha complicado por diferentes causas (Errasti, 2010): (a) estrategias de reducción del nivel de stock, (b) suministros más frecuentes y en menores cantidades, (c) reducción de los plazos de servicio en algunos sectores hasta las 24 y 48 horas a partir del stock disponible, (d) aumento en el número y variedad de referencias de producto, así como de canales de distribución con altos grados de exigencia en el nivel de servicio y (e) centralización del stock en los almacenes y conversión de almacenes regionales en centros de reexpedición con aumento de la criticidad de disponibilidad del stock. Esta mayor complejidad dificulta la mejora del desempeño logístico de los centros de almacenaje y distribución (Rushton et al, 2006), principalmente en lo que respecta a la gestión de stocks. Es necesario entonces, pronosticar la demanda de manera fiable para lograr bajos niveles de inventario de producto terminado, y proporcionar un mejor nivel de servicio al cliente (Buffa and Miller, 1979; Hax and Candea, 1984; Silver et al, 1998), y adicionalmente contar con herramientas computacionales avanzadas para que los responsables de aprovisionamiento logren hacer frente a este reto. Dentro de estas herramientas computacionales avanzadas se encuentran los sistemas expertos, 
creados a mediados de los sesenta por la comunidad de Inteligencia Artificial. La idea básica detrás de estos sistemas es la trasferencia de la experiencia humana a aplicaciones computacionales, con el fin de funcionar de la misma manera que lo haría un consultor. Esta tecnología proporciona soluciones a problemas específicos, siendo capaz de explicar la lógica que hay detrás de los resultados (Turban, E., \& Aronson, J. E. 200I)

Liu (1995) propone un sistema experto para la gestión de inventarios que combina cuatro módulos ( 1 ) clasificación $A B C$ de referencias, (2) elección del mejor método cuantitativo de previsión de demanda, (3) cálculo del lote económico y (4) cálculo del punto de pedido. Como Liu, otros autores como Tiacci (2009) y Tratar (2010) señalan la importancia de relacionar las previsiones de demanda con las políticas de aprovisionamiento empleadas. Dicen que aunque las previsiones sean utilizadas como una variable de entrada a los sistemas de gestión de inventario, la interacción entre ellos es generalmente ignorada.

Por otra parte, a pesar de haber disponibles en el mercado una gran variedad de sistemas comerciales especializados en la gestión de referencias, debido a su coste o a su complejidad de uso, solamente un porcentaje muy reducido de empresas los utilizan (Errasti 2009).

En este contexto, el presente artículo propone un sistema experto para la mejora de la gestión de inventarios SEMGI que pretende ser útil para asistir la toma de decisiones relacionadas al reaprovisionamiento de referencias gestionadas contra almacén, ayudando a reducir los niveles de inventario de producto terminado y mejorando el nivel de servicio brindado al cliente.

- Para su desarrollo se ha utilizado una metodología de investigación basada en un estudio de caso debido a su versatilidad para ser empleada tanto con fines exploratorios, como para construir, extender o probar una teoría (Rowley 2002, Yin 2009). Otros autores como Voss et al. (2002) también han señalado la importancia de la experimentación para los fines mencionados. El estudio de caso ha sido realizado en una empresa del sector de electrodomésticos de renombre a nivel mundial, donde el investigador no ha sido un simple observador, sino que ha estado involucrado en el proceso de cambio, propulsándolo con fines científicos. Se ha realizado un ciclo de mejora, para el cual el estudio de caso ha sido útil para cuantificar la utilidad del sistema, evaluarlo e identificar posibles mejoras. Para ello se han llevado adelante reuniones de Análisis de Valor o Value Analysis Workshop (VAW) con la contraparte de la empresa, conociendo en profundidad su modelo de negocio y logrando adaptar a éste el sistema experto desarrollado.

- La organización del artículo es la siguiente. En el apartado 2 se describen los cinco módulos del sistema experto, mientras que la sección 3 se focaliza en el caso de estudio realizado, donde se contextualiza la empresa seleccionada y las oportunidades de mejora allí identificadas. Finalmente, los apartados 4 y 5 contienen los resultados obtenidos y las conclusiones del análisis, respectivamente.

\section{Arquitectura del sistema experto SEMGI}

El sistema experto desarrollado (SEMGI) está compuesto por 5 módulos (ver Figura I): (I) genera una clasificación $A B C / X Y Z$ de las referencias según volumen y patrones de demanda, (2) calcula el stock de seguridad necesario para alcanzar el nivel de servicio objetivo, (3) selecciona el método de previsión de demanda que minimiza el error absoluto medio, (4) sugiere una política de reaprovisionamiento para cada subgrupo de productos y (5) monitoriza el sistema mediante el cálculo de una serie de indicadores de desempeño.

A continuación se presentan, según la literatura, los aspectos contemplados en los 5 módulos de la herramienta propuesta. Una vez detallados, se procede a la descripción del modo de funcionamiento del sistema en el apartado 2.5 .

\subsection{Clasificación de referencias}

La clasificación $A B C / X Y Z$ es una extensión de la estratificación $A B C$ clásica propuesta por Pareto. Este método toma en consideración no sólo el valor, sino también la variación de la demanda. Las referencias $X$ se caracterizan por un coeficiente de variabilidad bajo, las $Y$ por uno medio y las $Z$ por uno elevado (Reiner G. et al 2004). Esta herramienta puede ser utilizada para el control del inventario y para generar estrategias de aprovisionamiento. Según Rushton (2006) una estratificación de productos resulta útil desde el punto de vista logístico para mejorar al almacenamiento ya que permite establecer diferentes políticas para cada categoría de artículo. 
Figura |

\section{Esquema conceptual del sistema experto SEMGI}

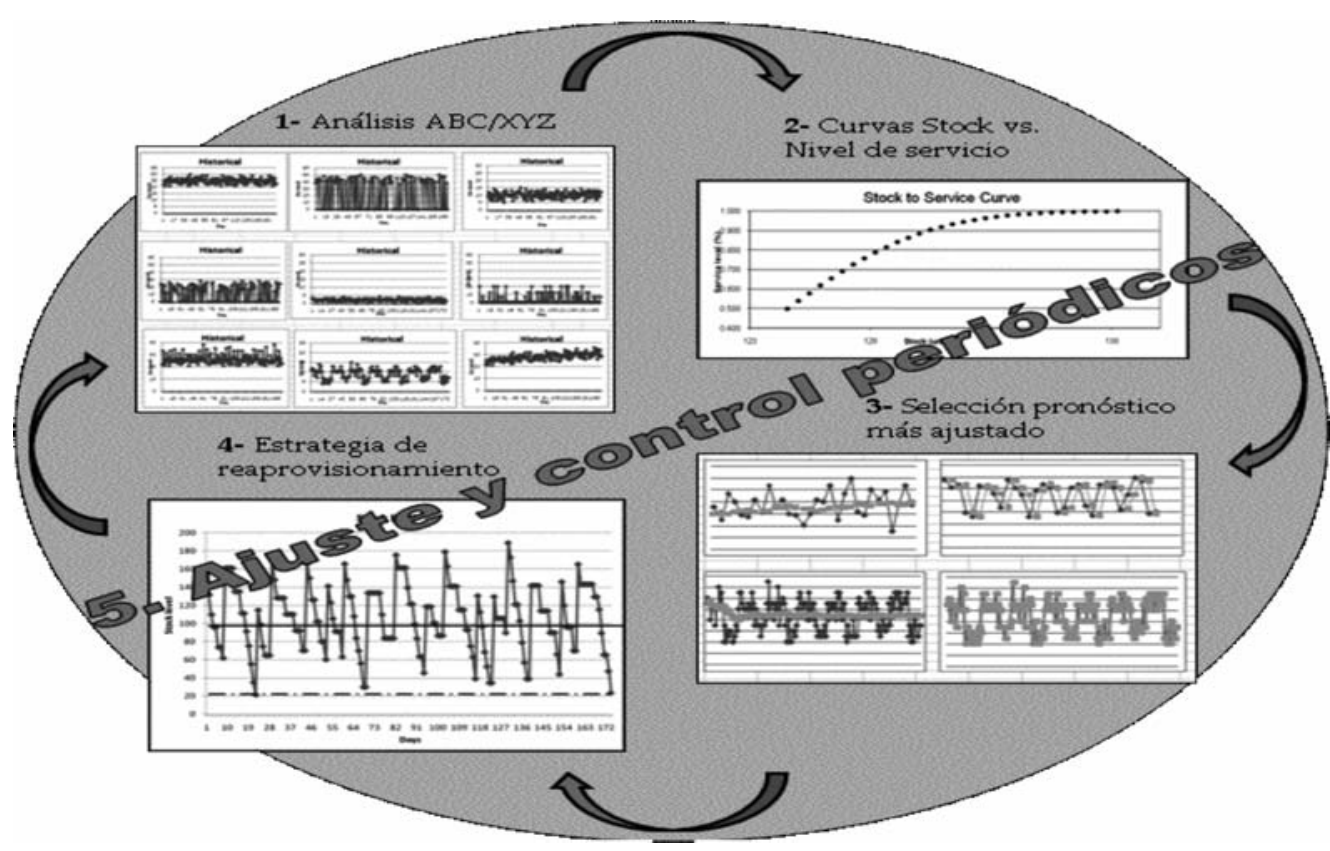

\subsection{Nivel de servicio y stock de seguridad}

Las políticas de planificación del servicio están íntimamente ligadas a las políticas de control de stock que se decida implantar (Sabriá 2003). El método de Gauss simplificado relaciona el stock de seguridad (SS) con el nivel de servicio que se brinda al cliente en función de Z, la variable normal tipificada [I].

$$
S S=Z^{*} D T^{*} \sqrt{P E}
$$

Donde DT representa la desviación típica de la demanda, PE el plazo de entrega del proveedor y los valores de $Z$ para cada nivel de servicio se obtienen en la tabla de distribución normal.

Conocer esta relación es de gran importancia para saber el coste del nivel de servicio con el que se pretende servir al cliente. Este cálculo genera las denominadas curvas Stock to Service (STS), que enseñan el incremento de stock necesario para aumentar el nivel de servicio actual.

\subsection{Previsión de demanda}

Se han desarrollado diversos métodos para pronosticar la demanda, algunos basados simplemente en registros históricos y en reglas estadísticas, mientras que otros apelan a la experiencia humana utilizando el juicio como principal herramienta (Webby and O'Connor 1996). Dentro del primer grupo, también conocidos como métodos cuantitativos de previsión, se despliega un abanico de previsiones de todos los grados de complejidad. Sin embargo, es necesario encontrar un equilibrio entre la complejidad del mismo y su bondad de ajuste. Puede darse que un modelo complejo con muy buenos ajustes produzca errores mayores que un modelo más sencillo y con un peor ajuste (Poler 2009). Esta paradoja, en conjunto con los resultados del estudio de Armstrong (2005), sugiere que pronosticar la demanda extrapolando los datos históricos con series temporales resulta apropiado.

Adicionalmente, existen herramientas que seleccionan el mejor método de previsión de manera automática, basadas en su mayoría en el cálculo del error. La medición de los errores cometidos en la previsión se realiza con variados objetivos, entre los cuales se destacan la comparación de diferentes modelos de previsión frente a una serie temporal y el seguimiento del ajuste de un método con el paso del tiempo (Poler 2009).

\subsection{Estrategias de aprovisionamiento}

Según Lewis (1970) existen básicamente dos grupos de políticas de aprovisionamiento; las basadas en el 
punto de pedido y un tiempo variable (SRC) y las que se sustentan en un periodo de revisión fijo y una cantidad variable hasta alcanzar un nivel de stock máximo (SRP). Entre estas dos categorías hay otras estrategias que introducen diversas modificaciones que combinan las características de las políticas puras. Liu (1995) menciona las siguientes: (I) (s,S), una variante del SRP, dónde un pedido variable es lanzado cuando al revisar el nivel de stock, el mismo se encuentra por debajo de una cantidad s y (2) un método combinado que propone la realización de pedidos de manera periódica y una vez se haya alcanzado el punto de pedido. Las órdenes lanzadas dentro de los intervalos fijos de revisión son de tamaño variable hasta alcanzar el stock máximo S, mientras que en las otras se pide una cantidad fija.

\subsection{Operativa o modo de funcionamiento}

El primer módulo clasifica las referencias de acuerdo al análisis $A B C / X Y Z$ y en función de la información histórica de ventas de las mismas. Se utiliza una clasificación sustentada básicamente en dos criterios:

I. Contribución al consumo en valor o cantidad. Se realiza un análisis de Pareto por cantidad y se divide las referencias según contribuyan al 80\% (A), I 5\% (B) o 5\% (C) de las ventas en volumen (porcentajes modificables en función del modelo de negocio).

2. Tipo y complejidad de la demanda (XYZ). Si el consumo se realiza de forma regular se considera $X$, si presenta cierta tendencia o estacionalidad, ya sea mensual o semanal, se clasifica como Y, mientras que si la demanda aparece de forma irregular o incluso intermitente se denomina $Z$. Por lo tanto, esta clasificación no solamente considera la desviación de los datos, sino que también identifica patrones de comportamiento repetitivos en el consumo.

El segundo módulo calcula el nivel de stock de seguridad necesario para alcanzar el nivel de servicio que se desea dar al cliente empleando la relación ya mostrada en la ecuación I.

El tercer módulo selecciona la técnica de previsión de series temporales (suavizado exponencial, medias móviles, media aritmética y último valor) más ajustado para cada clase ABC/XYZ en función del cálculo del error MAD, o mean absolute deviation. Se utiliza para ello un sistema de horizonte rodante, donde se simula que no se tiene el último dato real, se cal- cula su previsión y se obtiene el error de la diferencia en valor absoluto de estas dos cifras. El MAD es el promedio de los errores en un determinado periodo de tiempo. Si bien el error puede determinarse de diferentes maneras, se ha elegido el MAD por su facilidad de comprensión y utilización.

El cuarto módulo propone una estrategia de aprovisionamiento haciendo uso del método de previsión elegido. Se tienen en cuenta las estrategias puras tradicionales (sistema de revisión continua SRC y sistema de revisión periódica SRP), como también modificaciones de las mismas contemplando el consumo medio y la máxima demanda registrada en el periodo.

Finalmente, el quinto módulo evalúa el desempeño del sistema mediante el cálculo de una serie de indicadores (número de roturas, rotación del stock, recuperación del servicio, nivel de cobertura, nivel de stock medio y nivel de servicio).

\section{Estudio de caso}

El estudio de caso se ha llevado adelante en una empresa dedicada a la fabricación y distribución de electrodomésticos de línea blanca y pequeños electrodomésticos. Forma parte de un grupo europeo líder, que a nivel mundial ha logrado unas ventas anuales que rondan los 8.000 millones de euros. Posee una amplia cuota de mercado, ofreciendo diferentes gamas de productos, manejando una cantidad superior a las 2.000 referencias. El estudio se ha centrado en una plataforma de distribución regional, donde se han seleccionado artículos de diversas familias gestionadas contra almacén de producto terminado. Se ha seleccionado dicha compañía debido a su alta complejidad en gestión de inventarios, respondiendo a su vez a la problemática presentada en la introducción de este trabajo. Adicionalmente es una compañía reconocida en el sector por haber desarrollado un modelo de gestión de cadena de suministro referente con mejores prácticas en gestión de inventarios.

\section{I. Oportunidades de mejora}

El equipo investigador evaluó el sistema de gestión de inventarios empleado actualmente por la empresa identificando las oportunidades de mejora, así como los beneficios esperados a priori. Para facilitar la comprensión por parte del lector, las recomendaciones se han estructurado según los módulos del sistema experto SEMGl. 


\section{Clasificación de referencias}

La empresa motivo del caso clasifica sus productos de acuerdo a un $A B C / X Y Z$, en la cual el $X Y Z$ se determina por el cálculo de la desviación estándar de la cantidad consumida y la volatilidad de la demanda diaria. El principal inconveniente que presenta esta clasificación es que se basa en la hipótesis de que todas las referencias tienen un comportamiento normal. Un supuesto de normalidad puede introducir en los cálculos errores importantes, por ejemplo en referencias que se consume de manera intermitente. Se propone un nuevo criterio de clasificación que no considera solamente la desviación estándar de la demanda, sino que también identifica patrones de comportamiento repetitivos en el consumo de los artículos. Dicha clasificación se sustenta básicamente en dos criterios:

I. Contribución al consumo en valor o cantidad. Se realiza un análisis de Pareto por cantidad y se divide las referencias según contribuyan al 80\% (A), I5\% (B) o 5\% (C) de las ventas en volumen (porcentajes modificables en función del modelo de negocio).

2. Tipo y complejidad de la demanda (XYZ). Si el consumo se realiza de forma regular se considera $X$, si presenta cierta tendencia o estacionalidad, ya sea mensual o semanal, se clasifica como Y, mientras que si la demanda aparece de forma irregular o intermitente se denomina Z. Para el último grupo se propone la subclasificación de la Tabla I.

Tabla I

Subclasificación de referencias Z

\begin{tabular}{|c|l|}
\hline Nombre & \multicolumn{1}{|c|}{ Descripción } \\
\hline$Z$ & $\begin{array}{l}\text { Demanda irregular que se consume de manera } \\
\text { continua }\end{array}$ \\
\hline$Z 1$ & $\begin{array}{l}\text { Demanda intermitente que se consume en mo- } \\
\text { mentos muy específicos (puntual) }\end{array}$ \\
\hline Z2 & $\begin{array}{l}\text { Demanda intermitente que se consume en can- } \\
\text { tidades «constantes» cada periodos de tiempo } \\
\text { regulares }\end{array}$ \\
\hline Z3 & $\begin{array}{l}\text { Demanda intermitente donde las cantidades } \\
\text { consumidas varían pero el tiempo entre ventas } \\
\text { es regular }\end{array}$ \\
\hline
\end{tabular}

Se definen cuatro parámetros para realizar la estratificación XYZ propuesta. Los mismos junto con la forma en que determinan la clase de referencia se muestran a en la Tabla 2.

1. Frecuencia de consumo (Fc): Porcentaje de días en los que hubo consumo dentro de un periodo de tiempo. Mide la regularidad del consumo.

2. Distancia entre consumos (Dc): Promedio del tiempo que pasa entre dos pedidos consecutivos. También mide la intermitencia de la demanda. La desviación estándar de este parámetro $\operatorname{desv}(D c)$ mide la regularidad del consumo, o sea, si se realizan pedidos espaciados en el tiempo con un determinado patrón o de manera totalmente irregular.

3. $\frac{\text { Cpmensual }}{\text { Cmmensual }}$ Relación entre consumo pico y consumo medio con agregación mensual. Mide estacionalidad y tendencia.

4. $\frac{\text { Cpsemanal }}{\text { Cmsemanal }}$ Relación entre consumo pico y consumo medio con agregación semanal. Mide regularidad y tendencia dentro del mes.

Tabla 2

Clasificación en función de los parámetros propuestos $\mathrm{XYZ}$

\begin{tabular}{|c|c|}
\hline $\mathrm{Fc} \uparrow \mathrm{Dc} \downarrow \operatorname{desv}(\mathrm{Dc}) \sim 0 \frac{\text { Cpsemanal }}{\text { Cmsemanal }} \sim 1$ & \\
\hline $\mathrm{Fc} \uparrow \mathrm{Dc} \downarrow \operatorname{desv}(\mathrm{Dc}) \sim 0 \frac{\text { Cpsemanal }}{\text { Cmsemanal }}>1$ & $\frac{1}{\text { Zirregular }}$ \\
\hline $\mathrm{Fc} \downarrow \mathrm{Dc} \uparrow \operatorname{desv}(\mathrm{Dc}) \sim 0 \frac{\text { Cpsemanal }}{\text { Cmsemanal }} \sim 1$ & $\underset{\text { Z2 Pedido periódico regular }}{1}$ \\
\hline $\mathrm{Fc} \downarrow \operatorname{Dc} \uparrow \operatorname{desv}(\mathrm{Dc}) \sim 0 \frac{\text { Cpsemanal }}{\text { Cmsemanal }}>1$ & Z3 Pedido periódico irregular \\
\hline $\mathrm{Fc} \downarrow \operatorname{Dc} \uparrow \operatorname{desv(Dc)} \uparrow \uparrow \frac{\text { Cpsemanal }}{\text { Cmsemanal }}>1$ & $\underset{\text { Z1 Pedido puntual }}{\stackrel{\|\|}{\longrightarrow}}$ \\
\hline $\begin{array}{l}\mathrm{Fc} \uparrow \mathrm{Dc} \downarrow \operatorname{desv}(\mathrm{Dc}) \sim 0 \\
\frac{\text { Cpsemanal }}{\text { Cmsemanal }} \quad \text { (dentrodel mes) }>>1\end{array}$ & Y tendencia dentro del mes \\
\hline $\mathrm{Fc} \uparrow \operatorname{Dc} \downarrow \operatorname{desv}(\mathrm{Dc}) \sim 0 \frac{\text { Cpmensual }}{\text { Cmmensual }}>>1$ & Yestacional o con tendencia \\
\hline
\end{tabular}

Nivel de servicio y stock de seguridad

En lo que concierne a este punto, la empresa motivo de estudio no calcula las gráficas Stock to Servi- 
ce (STS), por tanto simplemente conoce la relación entre el stock y el nivel de servicio a posteriori a través de indicadores. La herramienta desarrollada calcula y grafica las curvas STS. Las mismas servirán para determinar el nivel de stock necesario para alcanzar el nivel de servicio objetivo.

\section{Previsión de demanda}

Con respecto a los métodos de previsión, la empresa gestiona sus inventarios de manera ligada, por lo que los pedidos en firme son tenidos en cuenta a la hora de realizar los pedidos a proveedor. Para la demanda incierta, se utiliza la media diaria móvil de los consumos medios para una cantidad preestablecida de periodos. Pero no es recomendable que el horizonte temporal sea el mismo para todas las gamas de productos. Por ejemplo, para un artículo cuya demanda cambia rápidamente deben mirarse periodos cortos que reflejen el comportamiento actual del mismo, mientras que en otros casos será útil tener en cuenta un horizonte de tiempo más extenso. Dependiendo de las características de la demanda (es- tacionalidad, tendencia o intermitencia), el sistema experto selecciona una de las técnicas o métodos de previsión mostradas en la Tabla 3 , donde $\mathrm{S}_{\mathrm{i}}$ representa la demanda real para el periodo i y Si la previsión para el periodo $i$.

\section{Estrategia de aprovisionamiento}

La herramienta sugiere una de las siguientes estrategias de aprovisionamiento para cada categoría. Dichas estrategias tienen parámetros de cálculo basados en el método de previsión elegido (VerTabla 4).

Los autores proponen las dos últimas estrategias de aprovisionamiento (SRS y SRS'), que resultan más eficaces para las categorías de consumo bajo e irregular.

\section{Resultados}

Para seleccionar la mejor política de aprovisionamiento, se han simulado todas las estrategias para las diferentes referencias y se han calculado los indica-

Tabla 3

Métodos de previsión empleados con sus respectivos procesos de cálculo

\begin{tabular}{|c|c|c|}
\hline Denominación & \multicolumn{2}{|c|}{ Fórmula } \\
\hline $\begin{array}{l}\text { Suavizado Exponencial }(\alpha=0.1 \text {, } \\
0.3,0.6 \text { y } 0.9)\end{array}$ & $\hat{S}_{n+1}=\alpha \cdot S_{n}+(1-\alpha) \cdot \hat{S}_{n} \quad 0 \leq \alpha \leq 1$ & \\
\hline $\begin{array}{l}\text { Suavizado Exponencial con } \\
\text { tendencia }(\alpha=0.1,0.3,0.6 \text { y } 0.9)\end{array}$ & $\begin{array}{l}\hat{S}_{n+1}=\alpha \cdot S_{n}+(I-\alpha) \cdot \hat{S}_{n} \text { (previsión sin tendencia) } \\
T_{n+1}=\beta \cdot\left(\hat{S}_{n+1}-\hat{S}_{n}\right)+(I-\beta) \cdot T_{n} \text { (tendencia) } \\
\hat{S}_{n+1}=\hat{S}_{n+1}+\frac{T_{n+1}}{\alpha} \text { (previsión con tendencia) }\end{array}$ & $\begin{array}{r}0 \leq \alpha \leq 1 \\
0 \leq \alpha \leq 1\end{array}$ \\
\hline $\begin{array}{l}\text { Suavizado Exponencial con } \\
\text { estacionalidad } \\
(\alpha=0.1,0.3,0.6 \text { y } 0.9)\end{array}$ & $\begin{array}{l}S f_{j}=\frac{\sum_{\text {mesj }} S_{i}}{\sum_{\text {año }} S_{i}} \text { (factor de estacionalidad) } \\
S_{n} \sim=\frac{S_{n}}{S f_{j}}(\text { demanda desestacionalizada }) \\
\hat{S}_{n+1} \sim=\alpha \cdot S_{n} \sim+(1-\alpha) \cdot \hat{S}_{n} \sim \quad 0 \leq \alpha \leq 1 \\
\hat{S}_{n+1}=\hat{S}_{n+1} \sim . S f_{j}\end{array}$ & \\
\hline $\begin{array}{l}\text { Media móvil (con diferentes } \\
\text { períodos de agregación: una, dos } \\
\text { y cuatro semanas) }\end{array}$ & $\hat{S}_{n+1}=\sum_{i=n-t}^{n} S_{i}$ & \\
\hline Medida aritmética & $\hat{S}_{n+1}=\frac{\sum_{i=1}^{n} S_{i}}{n}$ & \\
\hline Último valor & $\hat{S}_{i+1}=S_{i}$ & \\
\hline
\end{tabular}


Tabla 4

Estrategias de aprovisionamiento comparadas en el sistema experto

\begin{tabular}{|c|c|}
\hline Política & Descripción \\
\hline SRP. Sistema de revisión periódica. & $\begin{array}{l}\text { Se define un periodo fijo de revisión y el pedido se } \\
\text { realiza para alcanzar un nivel de stock máximo (lote } \\
\text { variable). }\end{array}$ \\
\hline SRC. Sistema de revisión continua. & $\begin{array}{l}\text { Cuando se alcanza el punto de pedido se solicita un } \\
\text { lote fijo al proveedor. El tiempo entre pedidos por lo } \\
\text { tanto es variable en función del nivel de consumo. }\end{array}$ \\
\hline $\begin{array}{l}\text { SRC'. Sistema de revisión continua con } \\
\text { actualización de parámetros periódica. }\end{array}$ & $\begin{array}{l}\text { Cuando se alcanza el punto de pedido se solicita un } \\
\text { lote que se recalcula basado en la previsión. El tiem- } \\
\text { po entre pedidos por lo tanto es variable en función } \\
\text { del nivel de consumo. }\end{array}$ \\
\hline SRS. Sistema de revisión según salidas. & $\begin{array}{l}\text { Al producirse una salida de mercancía se reaprovi- } \\
\text { siona el consumo. En este caso tanto el lote como el } \\
\text { tiempo entre pedidos son variables. }\end{array}$ \\
\hline $\begin{array}{l}\text { SRS'. Sistema de revisión según salidas hasta } \\
\text { consumo máximo. }\end{array}$ & $\begin{array}{l}\text { Al producirse una salida de mercancía se reaprovi- } \\
\text { siona hasta el consumo máximo. En este caso tanto } \\
\text { el lote como el tiempo entre pedidos son variables. }\end{array}$ \\
\hline
\end{tabular}

dores de desempeño definidos en el quinto módulo: número de roturas (cuenta la cantidad de roturas que se produjeron en el periodo de tiempo analizado), rotación del stock (cociente entre el consumo durante un período, y el valor del inventario medio), recuperación del servicio (días que tarda el sistema en poder a dar servicio otra vez luego de una rotura de stock), nivel de cobertura (cociente entre el valor del inventario medio, y el valor del consumo medio durante un periodo), nivel de stock medio (promedio de los niveles de stock registrados durante un periodo), y nivel de servicio (medido en función de la cantidad de roturas de stock producidas en el periodo de análisis).

A modo de ejemplo en la Figura 2 a se observa el comportamiento de una referencia $B Z$ si se utiliza un SRC basado en consumos medios, mientras que en la Figura 2b se emplea un SRC según consumos pico.

En este caso, la utilización de los consumos máximos registrados para establecer una política de aprovisionamiento, implica la reducción del 100\% de las ro-

Figura $2 \mathrm{a}$

Comportamiento de una referencia BZ si se utiliza un SRC basado en consumos medios

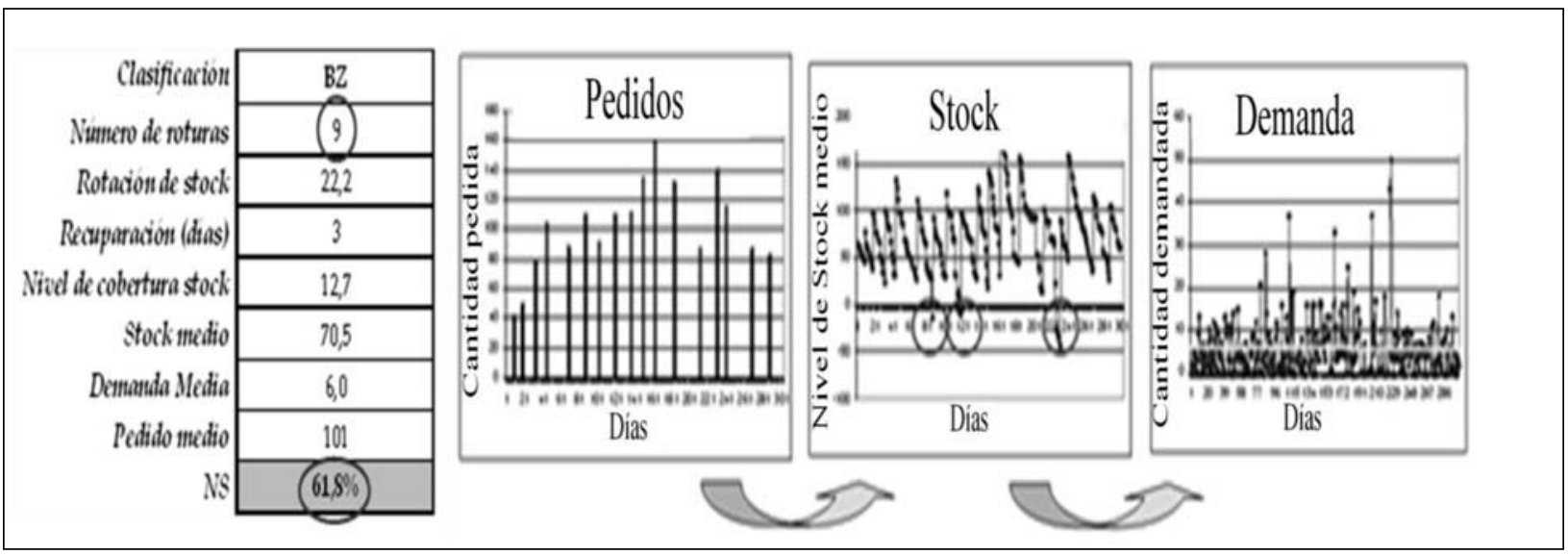


Figura $2 b$

Comportamiento de una referencia $B Z$ si se utiliza un SRC basado en consumos pico

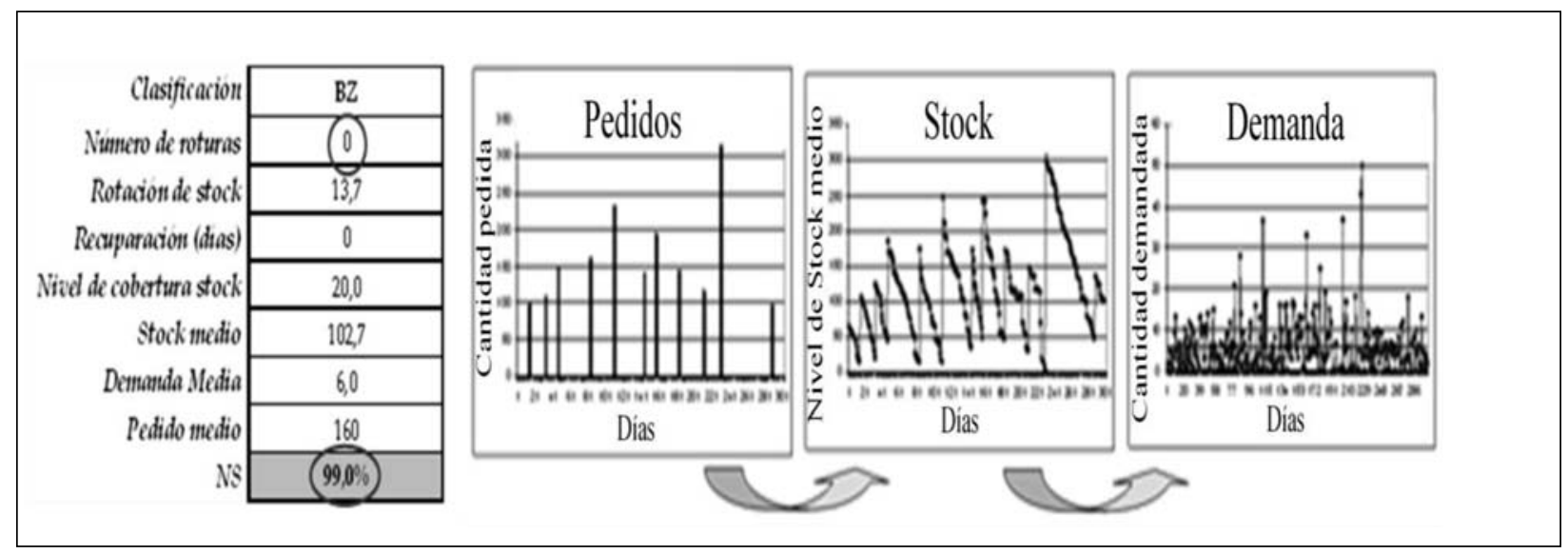

turas y el alcance del nivel de servicio objetivo. De la misma manera, para el resto de las categorías ABC/XYZ de productos, se compararon todas las estrategias mencionadas en el apartado anterior, apreciándose mejoras significativas al emplear unas u otras según el caso.

\section{I. Estrategias de aprovisionamiento y método de previsión por categoría $A B C / X Y Z$}

A continuación, la Tabla 5 resume las políticas de aprovisionamiento que suponen mejor rendimiento para cada grupo de referencias del caso de estudio. Adicionalmente se aprecian los métodos de previsión que generaron menor error durante la simulación en cada caso.

De acuerdo con la tabla, se recomienda el empleo de estrategias de aprovisionamiento con actualización periódica de parámetros en función del nivel de demanda, por su flexibilidad. Las mismas permiten adaptar la gestión a las fluctuaciones en los consumos registrados en el pasado cercano. Para referencias intermitentes Z2 y Z3, la utilización de sistemas de revisión según salidas permite brindar una adecuada respuesta sin necesidad de grandes niveles de stock.

Cabe destacar que la empresa no trabaja con artículos intermitentes de consumo medio o alto, por este motivo, no se observan resultados en lo que respecta a estas categorías.

\subsection{Resultados de desempeño}

En la Tabla 6 se sintetizan las características de la situación actual de la empresa, así como también la forma en la cual el sistema propuesto podría mejorarlas.

La implantación del sistema SEMGI por parte de la empresa permite alcanzar importantes mejoras en cuanto al nivel de stock de productos terminados de las categorías AX, BX y CX (más de un 30\% de reducción), manteniendo el nivel de servicio. Por otra parte, las nuevas estrategias de aprovisionamiento permiten incrementar el nivel de servicio en 4 puntos por encima del $90 \%$ en la categoría de artículos de consumo bajo e irregular, así como en referencias irregulares y con tendencia. La Tabla 7 resume las mejoras esperadas según la clasificación $A B C / X Y Z$.

El coste de inversión en software y tiempo de dedicación del equipo de proyecto tiene un retorno de inversión de 4 meses, solamente valorando la reducción del stock, y sin valorar el impacto del aumento del nivel de servicio.

\section{Conclusiones}

El artículo propone un nuevo sistema experto SEMGl para empresas con sistemas de producción contra almacén, que contempla de manera conjunta una categorización de referencias basada en patrones de demanda, técnicas de previsión de series temporales y la selección de estrategias de aprovisionamiento, simulando una política de servicio y controlando la gestión de inventarios en función del cálculo de indicadores de desempeño.

Permite una gestión semi-automatizada, por lo que la atención del planificador se centra en otras activi- 
Tabla 5

Estrategias de aprovisionamiento y mejores métodos de previsión de demanda según $A B C / X Y Z$

\begin{tabular}{|c|c|c|c|c|c|c|c|c|}
\hline & & X-Regular & Y: estacionalidad (S) & Y: tendencia $(\mathrm{T})$ & $\begin{array}{l}\text { Irregular } \\
\text { Z }\end{array}$ & $\mathrm{ZI}$ & $\begin{array}{l}\text { Intermitente } \\
\text { Z2 }\end{array}$ & Z3 \\
\hline \multirow{3}{*}{$\begin{array}{l}\text { A: } \\
\text { Alto }\end{array}$} & \multirow[b]{2}{*}{$\begin{array}{c}\text { Estrategia de } \\
\text { aprovisionamiento }\end{array}$} & SRC' & SRC' & SRC' & SRC' & $?$ & $?$ & $?$ \\
\hline & & $\begin{array}{l}\text { PP basado en el } \\
\text { pronóstico del } \\
\text { consumo medio. SS } \\
\text { según curvas ST }\end{array}$ & $\begin{array}{l}\text { PP según pronóstico } \\
\text { desestacionalizado } \\
\text { del consumo } \\
\text { medio. SS estacional }\end{array}$ & $\begin{array}{c}\text { PP según } \\
\text { pronóstico del } \\
\text { consumo medio. } \\
\text { SS con tendencia }\end{array}$ & $\begin{array}{l}\text { PP basado en el } \\
\text { pronóstico del } \\
\text { consumo pico. } \\
\text { SS según } \\
\text { consumo pico }\end{array}$ & & & \\
\hline & $\begin{array}{l}\text { Mejor método } \\
\text { de previsión }\end{array}$ & $\begin{array}{c}\text { Suavizado } \\
\text { exponencial con } \\
\text { factor de lisaje } \\
\text { mayor a 0,3 }\end{array}$ & $\begin{array}{l}\text { Suavizado } \\
\text { exponencial con } \\
\text { estacionalidad }\end{array}$ & $\begin{array}{l}\text { Suavizado } \\
\text { exponencial con } \\
\text { tendencia }\end{array}$ & \begin{tabular}{|c|} 
Suavizado \\
exponencial con factor \\
de lisaje \\
mayor a 0,3
\end{tabular} & & & \\
\hline \multirow{3}{*}{$\begin{array}{c}\text { B: } \\
\text { Medio }\end{array}$} & & SRC' & SRC' & SRC' & SRC' & $?$ & $?$ & $?$ \\
\hline & $\begin{array}{c}\text { Estrategia de } \\
\text { aprovisionamiento }\end{array}$ & $\begin{array}{l}\text { PP basado en el } \\
\text { pronóstico del } \\
\text { consumo medio. SS } \\
\text { según curvas STS }\end{array}$ & $\begin{array}{c}\text { PP según pronóstico } \\
\text { desestacionalizado del } \\
\text { consumo medio. SS } \\
\text { estacional }\end{array}$ & $\begin{array}{c}\text { PP según } \\
\text { pronóstico del } \\
\text { consumo medio. } \\
\text { SS con tendencia }\end{array}$ & $\begin{array}{l}\text { PP basado en el } \\
\text { pronóstico del } \\
\text { consumo pico. } \\
\text { SS según } \\
\text { consumo pico }\end{array}$ & & & \\
\hline & $\begin{array}{l}\text { Mejor método } \\
\text { de previsión }\end{array}$ & $\begin{array}{l}\text { Suavizado } \\
\text { exponencial con } \\
\text { factor de lisaje } \\
\text { mayor a } 0,3\end{array}$ & $\begin{array}{c}\text { Suavizado } \\
\text { exponencial con } \\
\text { estacionalidad }\end{array}$ & $\begin{array}{l}\text { Suavizado } \\
\text { exponencial con } \\
\text { tendencia }\end{array}$ & \begin{tabular}{|c|} 
Suavizado \\
exponencial con factor \\
de lisaje \\
mayor a 0,3
\end{tabular} & & & \\
\hline \multirow{3}{*}{$\begin{array}{c}\text { B: } \\
\text { Medio }\end{array}$} & & SRC' & SRC' & SRC' & SRC' & $?$ & $?$ & $?$ \\
\hline & $\begin{array}{c}\text { Estrategia de } \\
\text { aprovisionamiento }\end{array}$ & $\begin{array}{l}\text { PP basado en el } \\
\text { pronóstico del } \\
\text { consumo medio. SS } \\
\text { según curvas STS }\end{array}$ & $\begin{array}{c}\text { PP según pronóstico } \\
\text { desestacionalizado del } \\
\text { consumo medio. SS } \\
\text { estacional }\end{array}$ & $\begin{array}{c}\text { PP según } \\
\text { pronóstico del } \\
\text { consumo medio. } \\
\text { SS con tendencia }\end{array}$ & $\begin{array}{l}\text { PP basado en el } \\
\text { pronóstico del } \\
\text { consumo pico. } \\
\text { SS según } \\
\text { consumo pico }\end{array}$ & Identificar Outlier & $\begin{array}{l}\text { Se repone Qmed } \\
\text { al producirse una } \\
\text { salida. } S S=0\end{array}$ & $\begin{array}{c}\text { Se repone } \\
\text { hasta Qmax } \\
\text { al producirse una } \\
\text { salida. SS=0 }\end{array}$ \\
\hline & $\begin{array}{l}\text { Mejor método } \\
\text { de previsión }\end{array}$ & $\begin{array}{c}\text { Suavizado } \\
\text { exponencial o media } \\
\text { aritmética }\end{array}$ & $\begin{array}{c}\text { Suavizado } \\
\text { exponencial con } \\
\text { estacionalidad }\end{array}$ & $\begin{array}{c}\text { Suavizado } \\
\text { xponencial } \\
\text { con tendencia }\end{array}$ & $\begin{array}{c}\text { Suavizado } \\
\text { exponencial con } \\
\text { factor de lisaje } 0,1\end{array}$ & $\begin{array}{c}\text { Suavizado } \\
\text { exponencial o } \\
\text { media aritmética }\end{array}$ & $\begin{array}{c}\text { Suavizado } \\
\text { exponencial o } \\
\text { media aritmética }\end{array}$ & $\begin{array}{c}\text { Suavizado } \\
\text { exponencial o } \\
\text { media aritmética }\end{array}$ \\
\hline
\end{tabular}

Tabla 6

Resumen de las oportunidades de mejora detectadas en la evaluación del sistema

\begin{tabular}{|c|c|c|}
\hline & Situación actual & Mejoras planteadas por SEMGI \\
\hline $\begin{array}{l}\text { Clasificación } \\
\text { de referencias }\end{array}$ & $\begin{array}{l}\text { ABC/XYZ determinado por la cantidad consumida } \\
\text { y la variabilidad de la demanda según la desviación } \\
\text { estándar de la misma. Supuesto de normalidad que } \\
\text { en algunas referencias no se cumple. }\end{array}$ & $\begin{array}{l}\text { Nuevos criterios para clasificación } A B C / X Y Z \text { que } \\
\text { permiten identificar patrones de demanda } \\
\text { mediante el cálculo de una serie de parámetros: } \\
\text { (Fc, Dc, Cpsem/Cmsem, Cpmes/Cmmes). }\end{array}$ \\
\hline $\begin{array}{l}\text { Nivel de servicio } \\
\text { y stock de seguridad }\end{array}$ & $\begin{array}{l}\text { Dificultad para lograr la política de servicio y el ni- } \\
\text { vel de stock necesario. }\end{array}$ & $\begin{array}{c}\text { Cálculo del nivel de stock de seguridad necesario } \\
\text { para alcanzar el nivel de servicio objetivo en base a } \\
\text { simulaciones. }\end{array}$ \\
\hline Previsión de demanda & $\begin{array}{l}\text { Pronósticos con media móvil de los consumos } \\
\text { medios de una cantidad preestablecida de } \\
\text { períodos. Para referencias de consumo irregular } \\
\text { basarse en la demanda media no es suficiente. }\end{array}$ & $\begin{array}{c}\text { Elección entre diferentes métodos de previsión } \\
\text { con distintos horizontes temporales. Pronóstico de } \\
\text { la demanda pico para asegurar un nivel de servicio } \\
\text { adecuado, con un menor nivel de error. }\end{array}$ \\
\hline $\begin{array}{c}\text { Estrategia } \\
\text { de aprovisionamiento }\end{array}$ & $\begin{array}{l}\text { Sobre-stock en artículos de consumo regular, y } \\
\text { roturas en ferencias de consumo intermitente o } \\
\text { irregular. No se tiene una estrategia específica para } \\
\text { tratarlas ya que los patrones de demanda no se } \\
\text { asocian a la política de aprovisionamiento. }\end{array}$ & $\begin{array}{c}\text { Política de aprovisionamiento ligada a la } \\
\text { estratificación de referencias } A B C / X Y Z \text {; con } \\
\text { nuevas estrategias para artículos irregulares o } \\
\text { intermitentes y para aquellos de consumo regular. } \\
\text { Con una caracterización adecuada sería posible } \\
\text { una gestión semiautomática. }\end{array}$ \\
\hline
\end{tabular}


Tabla 7

Mejoras según clasificación $A B C / X Y Z$

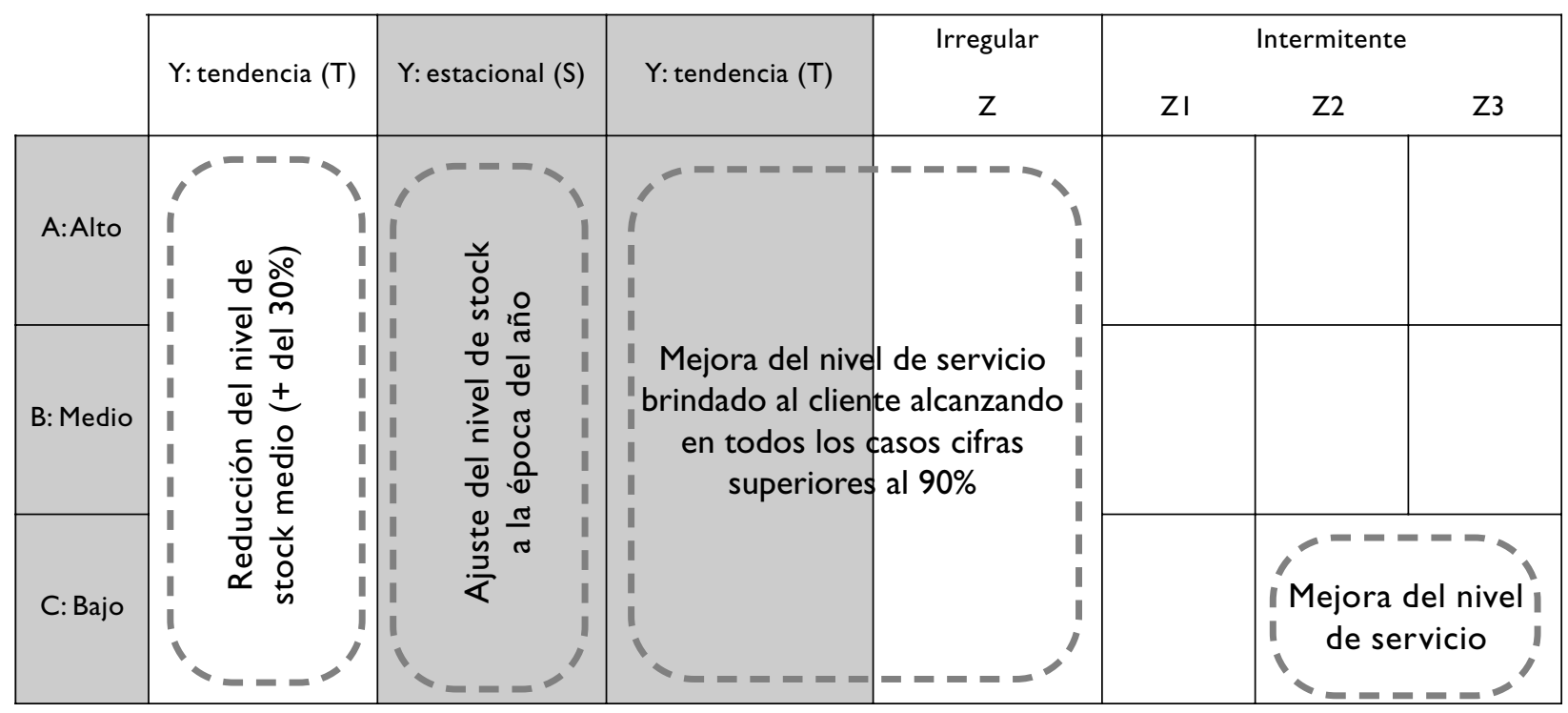

dades que agregan mayor valor para el cliente. Adicionalmente, se ha demostrado que proporciona un medio efectivo para la reducción de niveles de stock (más de un 30\% en referencias de consumo regular) y la mejora del nivel de servicio al cliente (brindando el servicio objetivo en artículos de difícil gestión).

Por todo lo expuesto, se concluye que SEMGl es una herramienta capaz de definir las la estrategia de aprovisionamiento idónea para cada categoría de artículos, garantizando un mejor rendimiento global de la gestión de inventarios y generando importantes reducciones de costes.

\section{Bibliografía}

ARMSTRONG J SCOTT, GREEN KESTEN C, (2005) Demand Forecasting: Evidence-based Methods, Strategic Marketing Management: A Business Process Approach, Ed. Luiz Moutinho and Geoff Southern.

BUFFA, E.S. y MILLER, J.G. (1979) Production Inventory Systems: Planning and Control. Third Edition. Homewood, IL, Irwin.

CHRISTOPHER MARTIN (2004) Logistics \& Supply Chain Management, creating value-adding networks, 3rd Edition, Pearson.

ERRASTI, A (2009) Proyecto: Sistemas de previsión de la demanda y su aplicación a la gestión de almacenes, Cluster de Transporte y Logística de Euskadi.

ERRASTI A, CHACKELSON C, ARCELUS M (2010) «Estado del arte y retos para la mejora de sistemas de pre- paración de pedidos en almacenes: Estudio Delphi», Dirección y Organización n40, 78-85.

HAX, AC y CANDEA, D ( 1984), Production and Inventory Management, Prentice Hall, Englewood Cliffs, NY.

LEWIS, C.D., ( 1970) Scientific Inventory Control, Butterworths, London.

LIU, C.Y.D. y RIDGWAY, K., ( 995$)$, «A computer-aided inventory management system - part I: forecasting» Integrated Manufacturing Systems, Vol. 6 No. I, pp. 12-21.

LIU C.Y.D. y KEITH RIDGWAY (1995), «A computer-aided inventory management system - part 2: inventory level control Reviews inventory policies and lot-sizing techniques in a cutting tool manufacturer» Integrated Manufacturing Systems, Vol. 6 No. 2, 1995, pp. II - 17.

POLER R, MULA J, PEIDRO D, (2009) «Determinación de parámetros de modelos de previsión de demanda a través de los errores de acierto en horizonte rodante», Dirección y Organización No. 37.

REINER G, TRCKA M, (2004) «Customized supply chain design: Problems and alternatives for a production company in the food industry. A simulation based analysis». Int. J. Production Economics 89 pp. 217-229.

ROWLEY JENNIFER (2002) «Using case studies in research». Manage Res News, 25(I).

RUSHTON,A., CROUCHER, P., BAKER, P. (2006). The handbook of logistics and distribution management, Kogan Page Publishers.

SABRIÁ, FEDERICO y colectivo (2003) La Cadena de Suministros- Editorial Gestiona. Logis. Book. 
SILVER, E.A., PYKE, D.F.y PETERSON, R. ( 1998). Inventory Management and Production Planning and Scheduling. John Wiley and Sons, Inc, New York.

TIACCI AND SAETTA (2009). «An approach to evaluate the impact of interaction between demand forecasting method and stock control policy on the inventory system performances». Int. J. Production Economics 118 pp. 63-71.

TRATAR, L.F., (20 I0), «Joint optimisation of demand forecasting and stock control parameters». International Journal of Production Economics doi:10.1016/j.ijpe. 2010.05 .009 .
TURBAN, E., y ARONSON, J. E. (200I). Decision support systems and intelligent systems, sixth Edition 6th ed. Hong Kong: Prentice International Hall.

VOSS C,TSIKRIKTSIS N, FROHLICH M. (2002) «Case research in operations management» Int J Oper Prod Manage; 22(2):195-219.

WEBBY y O'CONNOR, (I996) «Judgemental and statistical time series forecasting: a review of the literature» International Journal of Forecasting 12, pp. 91-। 18.

YIN R K (2009). Case Study Research Design and Methods, 4th edition, SAGE, US. 